\title{
«Die Hepatitis-Epidemie wurde jahrelang verschlafen»
}

\section{Bruno Kesseli}

Dr. med. et lic. phil., Chefredaktor

Philip Bruggmann, Chefarzt Innere Medizin der Arud Zentren für Suchtmedizin, gehört zu den Initianten des Netzwerks, das an einer schweizerischen HepatitisStrategie arbeitet (vgl. vorangehenden Artikel). Im Fokus des folgenden Interviews steht die Hepatitis C, gegen die keine Impfung zur Verfügung steht, die aber mit den neuesten antiviralen Medikamenten in über 90 Prozent der Fälle geheilt werden kann.

\section{Menschen mit einer chronischen Hepatitis C, eine lange vernachlässigte Patientengruppe, scheinen dank Ihres Netzwerks unversehens über eine starke, breit aufgestellte Lobby zu verfügen. Wie kam es dazu?}

In den letzten Jahren haben die Erkenntnisse zum Ausmass der Folgen der Hepatitis-Epidemie stark zugenommen. Getrieben von dieser Besorgnis einerseits und den ausbleibenden Reaktionen auf Seite der Gesundheitsbehörden anderseits hat sich auf Initiative der Schweizerischen Experten für Virushepatitis (SEVHep) das Netzwerk formiert. Die über 80 Persön-

\section{Philip Bruggmann}

PD Dr. med., Facharzt Innere Medizin, FMH. Chefarzt Innere Medizin der Arud Zentren für Suchtmedizin. Lehrarzt am Institut für Hausarztmedizin der Universität Zürich.

Arbeitsgebiet: Allgemeininternistische und infektiologische Versorgung von Patienten mit Suchtproblematik. Schwerpunkt: Hepatitis-C- und HIV-Versorgung. Mitarbeit in folgenden Kommissionen: International Network on Hepatitis in Substance Users (INHSU), SEVHep (Swiss Experts in Viral Hepatitis), SASL (Swiss Association for the Study of the Liver), Swiss Association for Medical Management of Substance Users (SAMMSU).
«Die Massnahmen können rasch umgesetzt werden»: Philip Bruggmann zur schweizerischen Hepatitis-Strategie. lichkeiten des Netzwerks kamen rasch zum Schluss, dass gehandelt werden muss.

Wie setzt sich das Netzwerk zusammen? Wer steht als treibende Kraft dahinter?

Das Netzwerk ist sehr breit abgestützt und umfasst alle Akteure, die mit viraler Hepatitis zu tun haben: neben Medizinerinnen und Medizinern und Betroffenen Politiker, Public-Health-Spezialisten, Forschende, Ethiker, Behörden. Vertreter der pharmazeutischen Industrie sind ebenso eingebunden wie auch Krankenversicherungen sowie Organisationen, die sich für Risikogrup-

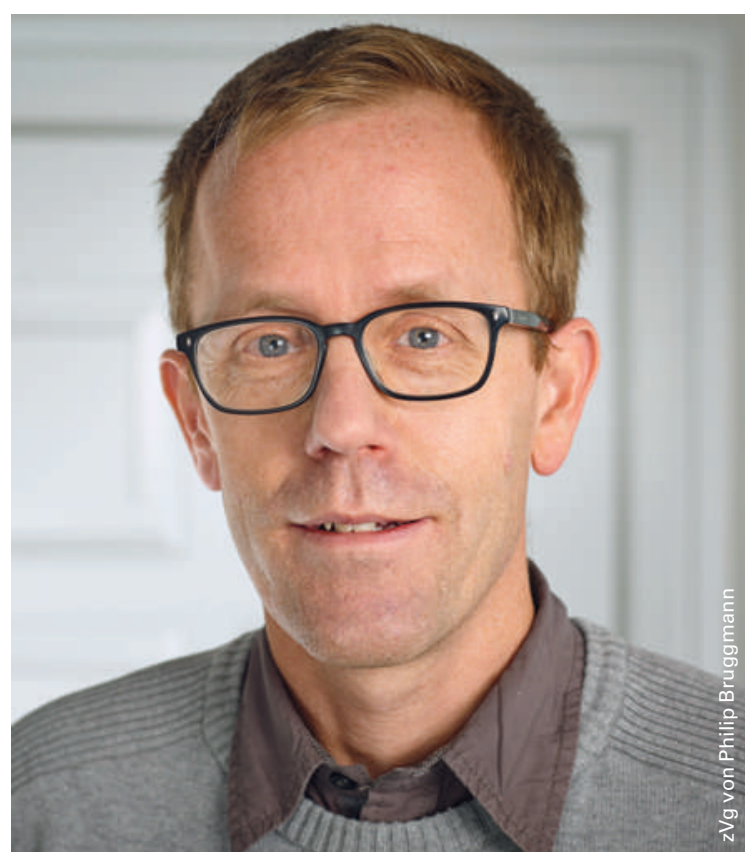


pen einsetzen. Diese Netzwerkmitglieder sind die treibende Kraft, die hochmotiviert und auf freiwilliger Basis an diesem Projekt mitarbeitet. Unterstützt werden die Bestrebungen von über 20 Institutionen und Gesellschaften aus allen Landesteilen, darunter Universitätskliniken, die Stiftung für Patientensicherheit, die Fachgesellschaften für Infektiologie, Hepatologie und Gastroenterologie sowie die World Hepatitis Alliance.

Die Hepatitis-Folgeerkrankungen nehmen zu, obwohl Hepatitis $\mathbf{C}$ heilbar ist und gegen Hepatitis B eine effiziente Impfung vorliegt.

In der Schweiz sind gemäss Bundesamt für Gesundheit 0,7 bis 1\% der Bevölkerung mit dem Hepatitis- $C$ Virus infiziert, weltweit $3 \%$. Weshalb braucht es eine "schweizerische Hepatitis-Strategie»?

In der Schweiz sterben seit mehreren Jahren mehr Personen an Hepatitis C als an HIV. Die Hepatitis-Folgeerkrankungen nehmen zu, obwohl Hepatitis $C$ heilbar ist und gegen Hepatitis B eine effiziente Impfung vorliegt. Mangelnde Aufklärung der Bevölkerung, ungenügendes Wissen auf allen Ebenen, eine schlechte landesweite Testperformance und tiefe Behandlungsraten sind die Ursachen dafür. Da diese Faktoren alle sehr eng miteinander verknüpft sind, müssen die Massnahmen dagegen gut aufeinander abgestimmt sein. Genau das bezweckt die Strategie.

Wie sehen die wichtigsten Pfeiler der Strategie aus? In sechs Arbeitsgruppen zu den Themen Prävention \& Aufklärung, Testen \& Überwachen, Zugang zur Therapie, Hochrisikogruppen, Finanzen \& Preise sowie Politik wird der jeweilige Bedarf erhoben und es werden Massnahmen geplant und zur Umsetzung gebracht. Ein zentrales Element der Strategie ist ihre Methodik: Dieselben Personen, nämlich das Netzwerk und daraus insbesondere die Arbeitsgruppen, die den Bedarf erheben und die Handlungsfelder definieren, koordinieren und planen in der Folge deren Umsetzung und stimmen diese mit den anderen Arbeitsgruppen ab. Dank des breit abgestützten Netzwerks tragen die Massnahmen und können sehr rasch umgesetzt werden.

Sie sprechen von einer Kluft zwischen der Bedeutung viraler Hepatitis für die öffentliche Gesundheit und den tatsächlich ergriffenen Massnahmen. Wie äussert sich diese Kluft?

Der Vergleich mit HIV veranschaulicht dies: Die Belastung von Hepatitis und HIV für die öffentliche Gesundheit ist ähnlich, bei den ergriffenen Massnahmen von
Seiten der Gesundheitsbehörden öffnet sich jedoch eine grosse Kluft. Im Bereich HIV ist die Schweiz dank der ergriffenen umfassenden Massnahmen ein Vorzeigeland bezüglich öffentlicher Wahrnehmung, Wissen und medizinischer Versorgung. Die HepatitisEpidemie, die schleichend und weniger dramatisch begonnen hat, wurde jahrelang verschlafen. So stellt heute die eigentlich heilbare Hepatitis-C-Infektion die Hauptursache für Lebertransplantationen dar. Das wäre nicht so gekommen, wenn rechtzeitig gehandelt worden wäre. Zudem: Mehr als die Hälfte der Betroffenen ist nicht getestet.

\section{Was braucht es, um die Strategie erfolgreich umsetzen zu können?}

Dazu braucht es eine enge Zusammenarbeit zwischen den nationalen und kantonalen Gesundheitsbehörden mit unserer privaten Initiative. Wir haben daher dem BAG eine Private-Public-Partnership vorgeschlagen. Das ist ein Novum im Bereich von Gesundheitsstrategien in der Schweiz. Angesichts des Spardrucks bei den Behörden einerseits und den Auswirkungen der Epidemie auf die Volkswirtschaft - der Grossteil der Hepatitis-C-Betroffenen ist im erwerbsfähigen Alter - drängt sich dieses Vorgehen auf, das übrigens auch von der Weltgesundheitsorganisation WHO empfohlen wird.

\section{In welchen Bereichen bestehen Hindernisse? Erleben Sie auch aktiven Widerstand?}

Die grössten Hindernisse sehe ich im Bereich des Wissens und der fehlenden Bekanntheit von viraler Hepatitis. So ist beispielsweise kaum bekannt, dass Hepatitis C nicht nur die Leber, sondern auch weitere Organe angreift. Nicht nur die leberbedingte Mortalität ist bei Betroffenen höher, sondern auch die Sterblichkeit aufgrund anderer Schädigungen durch Hepa-

\section{So ist beispielsweise kaum bekannt, dass}

Hepatitis C nicht nur die Leber, sondern auch weitere Organe angreift.

titis C. Hier herrscht viel Aufklärungsbedarf bei Behörden, Politikern und Krankenversicherern. Aber wir machen auch unter ärztlichen Kollegen, und dort insbesondere bei den für die Hepatitis-Versorgung so wichtigen Hausärzten, noch viel Aufholbedarf bezüglich Wissen, Testen und Weiterweisen an die behandelnden Spezialisten aus.

Wie ist der immer noch sehr hohe Preis der neuen Medikamente gegen das Hepatitis-C-Virus begründet? Für mich sind diese hohen Preise mit nichts zu begründen. Die Preise der neuen Hepatitis-C-Medikamente 
sind aktuell eine der grössten Hürden im Zugang zu einer adäquaten Versorgung der Betroffenen. Das ist sehr störend. Eine Ursache für die hohen Preise war, dass die herkömmliche Preisberechnung mit Ländervergleich und Innovationszuwachs, wie es unsere

Die neuen Hepatitis-C-Medikamente sind im Vergleich besser, einfacher und sicherer, womit kaum mehr Kontraindikationen bestehen.

Gesetze vorschreiben, zu kurz greift. Die bisherigen Interferon-basierten Therapien haben ihren Einsatz aufgrund des Nebenwirkungsprofils und der komplexen Anwendung selbst limitiert. Die neuen HepatitisC-Medikamente sind im Vergleich besser, einfacher und sicherer, womit kaum mehr Kontraindikationen bestehen. Der dadurch entstehende immense Zuwachs an Umsatzpotential hätte in die Preisberechnung wesentlich miteinbezogen werden sollen, was aber in der Schweiz nicht passiert ist. Stattdessen wurden medizinisch und ethisch bedenkliche Limitationen formuliert.

\section{Halten Sie es für wahrscheinlich, dass diese Medi- kamente in absehbarer Zukunft allen Patienten in der Schweiz zugänglich sein werden, die mit dem Hepatitis-C-Virus infiziert sind?}

$\mathrm{Ja}$, und das ist eines der Ziele der Strategie. Es bestehen vielversprechende Modelle von volumenbasierter Preisgestaltung im Ausland, beispielsweise in Australien, Italien und Portugal, die uns als Vorbild dienen. In Australien ist es gelungen, mittels eines solchen Preismodells den Anreiz für die Behandlungen anders zu setzen. Da die Pharmafirmen nur bis zu einem bestimmten jährlichen Volumen die volle Vergütung erhalten und der Preis pro Therapie nach Erreichen dieses Volumens kleiner wird, liegt der Anreiz für ein vermehrtes Behandeln bei den Ärzten und nicht mehr bei der Pharmaindustrie. Das ist ein sehr geschickter und richtiger Ansatz und nimmt den Kostendruck weg. So wird auch der Zugang für alle möglich: Die Behandlung in Australien ist seit dem 1. März dieses Jahres denn auch für alle unlimitiert zugänglich.

\section{Ist die "Vision" einer Hepatitis-C-freien Schweiz realistisch? Das Masernvirus gibt es hierzulande immer noch ...}

Unsere Vision, der Leitstern für die Strategie, ist die Elimination von Hepatitis B und C in der Schweiz. Mit Elimination ist per Definition nicht ein hepatitisfreies Land gemeint. Dies ist aufgrund der Migration und Reisetätigkeit unmöglich. Mit Elimination ist eine Reduktion der Inzidenz auf null als Resultat von be wusst ergriffenen Massnahmen definiert, fortgesetzte Kontroll- und Präventionsmassnahmen zur Verhinderung eines Neuausbruchs werden aber weiterhin nötig sein. Realistisches Ziel ist es, bis 2030 zumindest die Hepatitis-C-Situation auf Niveau von zum Beispiel Röteln zu bringen, für Hepatitis B wird es wohl etwas länger brauchen, da dort die Medikamentenentwicklung noch nicht so fortgeschrittenen ist wie bei Hepatitis C.

\section{Welche nächsten Schritte sind geplant?}

An der letzten Sitzung des gesamten Netzwerks der schweizerischen Hepatitis-Strategie wurden die Bereiche Aufklärung/Schulung, Testen und eine rasche enge Zusammenarbeit mit dem BAG als hoch prioritär erklärt. Wir planen eine erneute Kampagne rund um den Welt-Hepatitis-Tag am 28. Juli. Im Bereich Testen werden wir Massnahmen ergreifen sowie erweiterte Empfehlungen formulieren, um der grossen Testlücke zu begegnen. Mit dem BAG und dem EDI sind wir in engem

Unsere Vision, der Leitstern für die Strategie, ist die Elimination von Hepatitis $B$ und $C$ in der Schweiz.

Austausch. Weiter werden wir einen Vorschlag für eine innovative Preisgestaltung auf den Tisch legen, um aus der verfahrenen Situation zwischen dem BAG und den Pharmafirmen herauszukommen und um einen raschen freien Zugang zu den Medikamenten bei entsprechenden Preisanpassungen anzustreben. In etlichen Bereichen der Arbeitsgruppen laufen Studien und Erhebungen, aus deren Resultaten wir weitere Massnahmen ableiten werden. 